\title{
Cognitive Adhoc Routing Method Research Joint Study on Spectrum
}

\author{
Han Tianfeng \\ Qingdao Vocational and Technical College of Hotel Management, Qingdao \\ Shandong 266100, China \\ hantianfeng@126.com
}

\begin{abstract}
Cognitive radio technology free authorization through the use of opportunistic spectrum to improve spectrum utilization. Because in the cognitive wireless network, the communication between nodes must be without causing interference to the activities of the authorized user so you need to balance performance of cognitive wireless networks. This article puts forward a measurable spectrum joint routing algorithms, can in the spectrum sensing stage can't detect the level of the user's receiver to provide protection, and put onto a control channel routing information transmission, and from the perspective of energy efficiency design the routing discovery process, so that all in the middle of the forwarding nodes can get the spectrum or the network information, so in the destination node calculation consumption is also reduced.
\end{abstract}

Keywords: routing large-scale network, Congestion function, route discovery Cascading failure

\section{Introduction}

Routing metric, is choosing a path based on the parameters, namely choose the next hop node according to the standard. Choose the shortest distance, for example, the endto-end minimum hop count as a standard of route choice. Cognitive network because of its unique characteristics, some traditional routing metrics can no longer as the basis of the appropriate routing. Cognitive wireless Adhoc, define routing metrics should consider its own characteristics, a lot of research articles are aimed at this aspect, by combining the new characteristics of cognitive network, such as: the main users of channel using the model, cognitive users to main interference, the richness of cognitive resources available to the user, intermittent, cognitive user channel required by the cognitive users QoS (bandwidth, delay, throughput, etc.,) [1-2]. May interfere with the communication everywhere at the same time, a link in the interference within the scope of application of the same normal communication channel around the other link, interference can be a path within the mutual interference between each link (intra - flow interference), and also can be belong to different paths link between interference (Intel flow interference), and so on, will the above characteristics into consideration, to redefine the routing metric, the applications of these routing metrics to traditional routing algorithm (cases of distance vector routing algorithm, link-state routing algorithm), modified or agreement, for the traditional algorithm in order to achieve better end-to-end performance [3-5]. This article chose the AODV protocol modified to apply to cognitive wireless Ad hoc networks because in CRN, topology changes compared to the previous Ad hoc network has two parts, one is because of node mobility and the second is due to the mobility of the spectrum. Network topology of the frequent changes in the application of active routing will produce a large amount of 
overhead, but also consume a part of the network bandwidth, so the on-demand routing more appropriate than CRN. The most representative of on-demand routing protocol agreement as follows: AODV and DSR protocol. AODV is the routing protocol by jumping type, DSR is source routing protocols. Don't choose the DSR because the DSR is source routing protocols, intermediate nodes do not maintain routing information, and the main characteristics of the cognitive network is the intermittent frequency spectrum, the spectrum is unavailable causes the link is not available is relatively frequent link characteristics of small scale, the DSR cannot quick response to this change, may cause the routing to adopt, greatly increased the routing overhead. And AODV is by jumping mechanism, each intermediate node to maintain a routing table, routing information is advantageous to the small scale of change tracking, later in this article the reference in the design of routing protocol AODV routing protocol mechanism, so here for the first agreement in the cognitive radio environment problems were analyzed [6-8].

(1) the problem of data forwarding; Cognitive Ad hoc network is a multiband, a terminal (nodes) may have more than one channel can be used to transmit data, and routing algorithm should not only choose the next-hop node, and choose a channel of data transmission, due to the diversity of cognitive users to its spectrum, a user when forwarding data, when found its spectrum is unavailable, can wait for a period of time until the spectrum available again, you can also change the launch parameters, to forward data from another band, or can be applied at the same time a few repeated channel to transmit data in order to improve the accuracy of the data transmission. This led to some scheduling algorithm research; the scheduling is more business data, multiband scheduling problem. AODV failed to consider the aspect of it (2) the link and the asymmetry of the nodes; CRN each node has its own use of spectrum in the collection of the spectrum in the collection may set a few spectrum resources, at the same time, each node of the spectrum collection may be different, that is what we are defined by the spectrum, the spectrum difference within the scope of the node is not necessarily can spread even in communication. The spectrum in the CRN, each node use is likely to be different, that between nodes link carries different spectrum can lead to different link performance, so cases each link as symmetry is not reasonable. The AODV protocol is designed for single frequency network, the link between network nodes using the same frequency band, in that sense link and node is symmetric. (3) the spectrum utilization; Original AODV protocol is based on the point of single frequency network, routing problem completely reflect the choice of the next hop node, and cognition in the distributed network, the routing protocol design should not only consider the next-hop node selection, but also on the link and the next-hop choose which frequencies, while the spectrum available for CR users is discrete in time, and this may affect the quality of CR user's service, is due to this characteristic, the CR users can continue to receive data on a switch to the new spectrum, also can choose on several spectrum channel of the best quality as CR spectrum used by user, or transmission of the data flow and application of several channel (4) using the intermittent; CRN most essential characteristic is the use of spectrum is the main users of free frequencies, when the primary user occurs, CR users must immediately stop the use of the frequency band in place to ensure that does not affect the quality of authorized user's business. In CR users point of view, this channel is intermittent, CR of connections between nodes because of this nature also become intermittent connection, in the traditional Ad hoc networks, AODV protocol that all nodes can have been using spectrum, don't need to consider the spectrum used. But in cognitive distributed 
network, must consider the spectrum of intermittent problem, and how to choose a high stability of links. Two node link spectrums is unavailable due to breaking, is to continue to wait for the spectrum can be used to establish connections, or choose other spectrum, or choose other paths to complete end-to-end data transmission is a problem of routing protocol must be considered.

\section{Related Works}

(1) The path hop

The path of the end-to-end hop count as a kind of routing metrics is a simple and intuitive thinking. End-to-end hop count as a routing metric, which based on the basic thoughts of the wired network, there are two basic assumptions: or communication link between two nodes, or does not exist; in the network communication link has the same link quality. The above two conditions in the cable network is easy to implement, but in the wireless network communication environment, fading, and interference due to are uncertain, link can't be with the same quality. Wireless environment, the obvious: hop reduces each jump distance increases, the signal transmitted over long distances would lead to more serious wireless propagation loss, will be a little more than those on the hop path has a higher packet loss rate; In addition, even if a few paths have the same hop count, but the link between each jump will due to link interference, decline the end to-end performance of different, just define the routing metric for the hop, not specific reflect the path of those who have the same hop end-to-end performance difference.

(2) ETX

ETX (Expected Transmission Count) is the Expected number of Transmission for data Transmission. When the source node to destination node sends a packet, need intermediate nodes forward to get to the destination node. Ideally, the packet transmission is equal to the number on the path to the path hop. But link the unreliability of packet loss; in order to correctly receive packets need to be on a link in the packet retransmission for many times. We think the MAC layer successfully send a packet to the receiver receives the right, and returned to the sender a right to receive confirmation (ACK). If use $p_{f}$ and $p_{r}$ respectively link packet loss rate of the forward and reverse (no successful packet sending packets to total ratio), is not on a link in the probability $p$ of successful transmission of a packet is:

$$
p=1-\left(1-p_{f}\right)\left(1-p_{r}\right)
$$

Hypothesis cannot be retransmission; successful transmission of packets will be the probability of transmission time is:

$$
s(k)=p^{k-1}(1-p)
$$

Accumulative the required transmission times expected EXT for:

$$
E T X=\sum_{k=1}^{\infty} k * s(k)=\frac{1}{1-p}
$$

Measurement of the path is the path including the sum of each link ETX, routing algorithm to choose the path of least path metric (3), assuming each packet the packet loss rate is independent identically distributed. And the routing metric is a two-way street, and from node to node link from node to node and link routing metric is the same. Although ETX than minimum hop routing metrics can get better performance, because it takes into account the quality of the link, but in some situations still can't choose the 
optimal path, for example, in one with 802.11 a and $802.1 \mathrm{lb}$ of two kinds of interface nodes in the network, reference ETX routing will be more preferred $802.1 \mathrm{lb}$ link to transmit data, there are two main reasons for this: one is the ETX only consider link packet loss rate without considering their bandwidth, the second is the length of the path, the choice of ETX more give preference to the shorter path because of the shorter path retransmission times can be reduced, which leads to the two kinds of interface of the network, 802. The $11 \mathrm{~b}$ link would be more applications including, reduce the use of the channel at the same time increase the network congestion.

\section{(3) WCETT}

In order to optimize the ETX routing metric, the bandwidth is also considered, [9] proposed a new routing metric WCETT (Weighted Cumulative Expected Transmission Time), defined WCETT link on every success to send a packet Time expect ETT (Expected Transmission Time) is equal to:

$$
E T T=E T X \times \frac{S}{B}
$$

Where $\mathrm{S}$, the number of bits in the packet $\mathrm{B}$ for the link transmission rate (bandwidth), ETX for the total number of data packets. Suppose a path (path) has $n$ links, using $k$ channels, then the packet transmission on a path of total time for:

$$
W C E T T=\sum_{i=1}^{n} E T T_{i}
$$

Considering the influence of channel diversity, define another measure, the application of $j$ channel data transmission total time:

$$
X_{j}=\sum_{i \text { to } j} E T T_{i}
$$

A path of average throughput determined by bottleneck channel, so the definition

$$
W C E T T=\max _{i \leq j \leq k} X_{j}
$$

When finally define routing metrics, considering both the data transmission time, considering the diversity of the channel will lead to a path on the application of channel less flow caused by disturbance, thus affecting the end-to-end throughput:

$$
W C E T T=(1-\beta) * \sum_{i=1}^{n} E T T_{i}+\beta * \max _{i \leq j \leq k} X_{j}
$$

For $\beta$ in $[0,1]$ the variable weighting factor

The first item in the style reflects the end-to-end accumulative total transmission delay, assuming a path two link exists on the application of the same channel interference, and the second reflection is the packet in the path of the interference is the most serious of the sum of transmission time on the link., the greater $x_{j}$ the flow within the greater interference, and thus affects the throughput of the whole path. To sum up, the routing metric WCETT is top to reflect the path transmission delay and link interference of a kind of compromise, we should choose WCETT smaller path as a data transmission path. Weight coefficient is the origin of the adjustment $\beta$. When $\beta=0.9$, 
the more important paths of interference; When $=0.1$, more emphasis on the path of the end-to-end delay performance.

Whatever the routing metrics definition, when the system routing metric is defined as several separate routing metrics weighing your options, attention should be paid to the following:

First several routing metrics that regulated by the coefficient of less than 1 greater than 0 respectively in rodents by the proportion of measurement. Second, the several separate routing metric values range in the same order of magnitude as far as possible, if there are orders of magnitude difference should guarantee the order of magnitude of large number and fractional order of magnitude ratio must be less than 1000 .

Third, these routing metrics should be consistent with the increase and decrease, routing metrics is the basis of routing, these separate routing metric must keep consistent with routing metric system increase or decrease routing metric system can ensure to obtain the extremum.

The understanding of the routing metric design criteria for design laid the foundation of cognitive network routing metric.

\section{System Model Description}

\subsection{Problem Posing}

$\mathrm{CR}$ (cognitive radio, cognitive radio) as a new intelligent wireless technology, make SU (secondary users, users) by detecting the PU (primary users, primary user) idle spectrum, in does not affect the work authorized users under the premise of smart to choose and use the idle spectrum, thus greatly improve the spectrum utilization of existing inefficient. At present to $\mathrm{CRN}$ (cognitive radio networks, cognitive radio networks) research focuses on spectrum sensing and spectrum management, CRN routing algorithm of design is still a problem needs to be further studied. Traditional Adhoc applications is a fixed frequency band, the routing protocol design main consideration is the node movement brought about by the problem of dynamic network topology, the research of this kind of routing algorithm are relatively common. In the cognitive wireless Ad hoc networks, due to the diversity of the spectrum used by each SU set, time-varying and differences, in traditional Adhoc routing metric is no longer applicable. Definition and selection of the routing metric largely should consider the main users of channel using the model, at the same time, in order to make the user data reliable transmission, mutual interference between users should also be considered.

\subsection{Models and Assumptions}

In cognitive wireless Ad Hoc networks, PU in authorized spectrum communications, SU with an opportunity to use authorized users access to the spectrum, namely a certain period of time, SU can use the user does not use the frequency band. In a network of SU by jumping more communicate, each $\mathrm{SU}$ has spectrum detection ability, can detect the primary users in a certain period of time does not use the entire spectrum, to choose their own access. Assumes that the primary users are independent of each other between each channel, and the ON/OFF random process, using the model in the main user channel, when the channel in a state of SU can use the channel. Said $\left\{N_{n}, n \geq 1\right\}$ in the open State (ON - SMe) sequence of random variables, $\left\{F_{n}, n \geq 1\right\}$ said in a closed State (OFF - State) sequence of random variables, are the two random variables sequences 
are two counting process. Assume that the two sequences of independent random variables and to obey the strength $n(t)_{i}$ and $f(t)_{i}$ the poisson process respectively, the nonhomogeneous poisson process (parameters related to time) can be converted into homogeneous poisson process is analyzed, so for a period of time, the two random variables to obey $n(t)_{i}$ and $f(t)_{i}$ homogeneous poisson distribution. Each section of the duration $(0, t]$ of the ON and OFF state $n_{i}$ and $f_{i}$, respectively, to obey the parameters $\xi_{n}$ and $\zeta_{n}$ exponential distribution. So over a period of time $\lambda_{i} \mu_{i}$, the main users of sum of time $(0, t]$ :

$$
Y_{t}=\sum_{n=1}^{N_{t}} \xi_{n}
$$

Users can use time sum as follows:

$$
S_{t}=\sum_{n=1}^{F_{t}} \zeta_{n}
$$

Both for the compound poisson process, for the user, the utilization rate of channel $\mathrm{i}$ as follows:

$$
U_{i}=\frac{E\left(S_{t}\right)}{E\left(S_{t}\right)+E\left(Y_{t}\right)}=\frac{f_{i} \lambda_{i}}{f_{i} \lambda_{i}+n_{i} \mu_{i}}
$$

\subsection{The Routing Metric Design}

(1) In the cognitive wireless Ad Hoc networks, SU called cognitive nodes, cognitive between nodes communicate by way of self-organization. A cognitive network available in the main user channel number to see if the two cognitive nodes can communicate, according to its links. The source node to the destination node of a path containing $\mathrm{P}$ link, channel $i, i \in N$ link its link throughput for application:

$$
R_{k}=W_{i} \log _{2}\left(1+\frac{p_{k}}{d_{k}^{n} N_{0} W_{i}}\right)
$$

One $W_{i}$ for the bandwidth of the channel i, to the length of the link. For the power of the sending node for gaussian noise power spectral density, as the path loss factor.

Because the channel is given priority to the user's authorization frequencies, for the user, the throughput of the link:

$$
r_{k}=U_{i} \times R_{k}
$$

For $U_{i}$ the channel utilization.

A path containing $\mathrm{P}$ link, so the average throughput of this path:

$$
A T=\frac{1}{P} \sum_{k=1}^{P} r_{k}
$$


(2) To make cognitive node data reliable transmission, to take into consideration the disturbance of other cognitive nodes to it, the letter to noise ratio. Define $k$ the sending node on even the road is $k$, the receiving node $k+1$, the received power is:

$$
p_{k+1}=\frac{p_{k} G_{k} G_{k+1}\left(h_{k}^{2} h_{k+1}^{2}\right)}{d_{k}^{n} L}
$$

Parameters for the system loss $L$ and for k node antenna gain $G_{k}$, antenna height, for $\mathrm{k}$ node $h_{k}$. Because each cognitive node in distributed cognitive network are in equal status, namely each cognitive nodes have the same transmission power, firing distance, interfere with the parameters such as distance, so (15) can be simplified as:

$$
p_{k+1}=\frac{p G^{2} h^{4}}{d_{k}^{n} L}
$$

When not on the same path and the interference within a distance of two nodes using the same channel, will interfere with each other. For $k+1$ node application channel I suppose interference node for $\mathrm{j}$, for $\mathrm{k}+1, \mathrm{j}+1$ node interference power is:

$$
I_{j, k+1}=\frac{p G^{2} h^{4}}{d_{j, k+1}^{n} L}
$$

One $d_{j, k+1}$ for $\mathrm{j}$ node to $\mathrm{k}+1$.

According to the concept of the [4], for a period of time $(0, t]$, to use the channel i $\mathrm{k}+$ 1 nodes, the number of the interference sources $\mathrm{M} \lambda_{j i}$ obeying poisson distribution, namely:

$$
B\{j(t)=M\}=\frac{\left(\lambda_{j i} t\right)^{M} e^{-\lambda_{j i} t}}{M !}
$$

So the total interference power is:

$$
I_{k+1}=\sum_{j=1}^{M} I_{j, k+1}
$$

Define k links dry noise ratio:

$$
\operatorname{SINR}_{k}=\frac{p_{k+1}}{I_{k+1}+N_{0} W}
$$

After $\mathrm{k}$ link, define the unit of data transmission correct probability as follows:

At that time $\operatorname{SIN} R_{k} \geq 10$, the correct probability of link k for $P_{c}(k)=1$;

At that time $\operatorname{SIN}_{k}<1$, the correct probability of link for $P_{c}(k)=0$;

At that time, $1 \leq \operatorname{SIN}_{k}<10$ link the correct probability of $P_{c}(k)=\operatorname{SIN} R_{k} / 10$.

$\mathrm{P}$ the correct probability of paths available:

$$
\prod_{K=1}^{P} P_{c}(k)
$$


To unit data at the destination node error-free receiving, define the transmission times $1 / P_{c}$.

As finally determined by the worst link quality of path, so define the drying ratio as follows:

$$
\operatorname{SIN} R=\min _{k \in P}\left\{S I N R_{k}\right\}
$$

(3) for any A link, which channel, regardless of its use of the channel to use time expectations for [10] has been proved that the bigger the expectation was confined to let state transitions between the slower, define the EAT (expectation of the available time, the expectations of available time) :

$$
E A T=\min _{k \in P}\left\{1 / \mu_{k}\right\}
$$

Finally considering trade-off between the above a few parameters, the definition:

$$
A E S=((1-\beta) A T+\beta E A T)(\operatorname{SINR})^{\alpha}
$$

Among them $\alpha$ and $\beta$ all are weighting factor, and have $0 \leq \alpha \leq 1,0 \leq \beta \leq 1$.

\section{Simulation Results and Analysis}

Is a dedicated network simulation software NS2, OPNET, GloMosim, MATLAB, etc., NS2 (Network simulator) is open source components, protocols, rich, strong expansibility and result credibility higher characteristic, and of its protocol component $\mathrm{C}$ code with real-world code close to agreement, agreed to reduce secondary let hair transplantation in real system, making it especially suitable for colleges and universities for research and teaching. OPNET (Optimized Performance Network Engineering Tool) as commercial software has rich component, friendly interface and automatic analysis, etc. But because it is expensive, commercial software don't facilitate normal scientific research institutions and researchers use, open difference makes it hard for its research and extension. GloMosim (Global Mobile Information System simulator) is developed by the University of California, Los Angeles and large-scale network simulation platform for wireless network System. It is written in a Parsec parallel computing language. GloMosim has higher efficiency, better expansibility and usability. GloMosim hierarchical structure is too strict, however, is not conducive to the cross layer to access support, and due to the dedicated to the wireless network simulation, so the scope of application and popularity isn't big NS2. MATLAB (MATrix LABoratory) is introduced by the American Mathworks company system used for the graphics and scientific computing environment. The environment for users to integrate the process of numerical calculation and graph drawing, file management, artificial intelligence, neural network research in the field of industrial control and other functions. MATLAB is a excellent mathematical application software, as a network simulation software in the efficiency and applicability have obvious flaws, such as more suitable as a result of the validation of association analysis simulation tools. Wireless module was originally introduced by CMU Monarch of the working group to NS, the wireless module is the core of mobile node (MobileNode), mobile node is on the basis of basic nodes to join some function of wireless mobile nodes need to constitute, includes Network components of the Link Layer, Link Layer, LL), ARP module, Interface Queue (Interface Queue, IFq), MAC Layer and Network Interface (Network Interface). Mobile 
node is through the network interface to connect to the wireless Channel (Channel). Node itself and unicast and multicast nodes, this paper studies the routing protocol is unicast routing protocols. Mobile node can move within a given topology, make use of the signal is received or transmitted in wireless channels. Through above the node load all kinds of Agent (Agent), or set the various properties of node, to complete the different functions. The characteristics of the mobile node makes the NS2 to simulate multiple hops Ad hoc network, wireless LAN and wireless environment. Is needed in NS2 simulation of wireless protocols and algorithms, all you need to write into the corresponding network level agents, binding and mobile node before you run.

Simulation parameters set: in the area of $10 \mathrm{~m} \times 10 \mathrm{~m}$, randomly distributed 10 cognitive nodes, source nodes coordinates $(0,0)$ and destination node coordinates of $(10,10)$. Launch distance of each node is 6 meters; interference distance is twice the transmission distance, commonly defined as 12 meters. Transmission power $\mathrm{P}=$ $1 \mathrm{mWW} / \mathrm{Hz}$, main user channel bandwidth $\mathrm{W}=15 \mathrm{~Hz}$.

The simulation steps:

Step one: in the area of 10 meters xlO meters, eight cognitive nodes randomly distributed, is limited to 7 jumps, randomly selected three source node to the destination node path.

Step 2: respectively by M / / (minimum hop, minimum hop), AT the SINR, and for routing metrics, respectively from the three path chosen make ats, SINR, maximum, minimum "optimal" path.

Step 3: choose the path are calculated separately, and the average throughput, delay, the correct probability and the number of retransmission. The basic unit of average throughput is bits/SEC, normalization of time delay is a bit of data transmission time on the path.

We will repeat the experiment 30 times, and finally take their average value simulation results are obtained.

Figure 1, Figure 2 and Figure 3 respectively applied the end-to-end performance of different routing metrics chosen path. In Figure 1, because the AT routing metrics consider link throughput routing, its maximum throughput. And $\mathrm{MH}$ with minimum jump to measure did not consider the status of the link, its minimum throughput. Considering various factors weigh, and its throughput is very big also. Figure 2 shows the end-to-end performance of normalized time delay, the AT los largest path selected by measuring the average throughput, its minimum delay. Choose the minimum hop road, time delay is small. AES, considering various factors weigh because it is over, the time delay between the two. Did not consider the factor of any delay so delay is the largest. Figure 3 units receives the correct probability of a data, SINR routing metrics consider letter to noise ratio, by the path of the unit for the data AT the destination node receives the correct probability of the largest, $\mathrm{MH}$ and signal-to-noise ratio (SNR) AT does not take into account any relations and accuracy is very small. Comprehensive these simulation results can be seen, due to the AES considering various factors, its end-to-end performance is relatively good. 
International Journal of Future Generation Communication and Networking

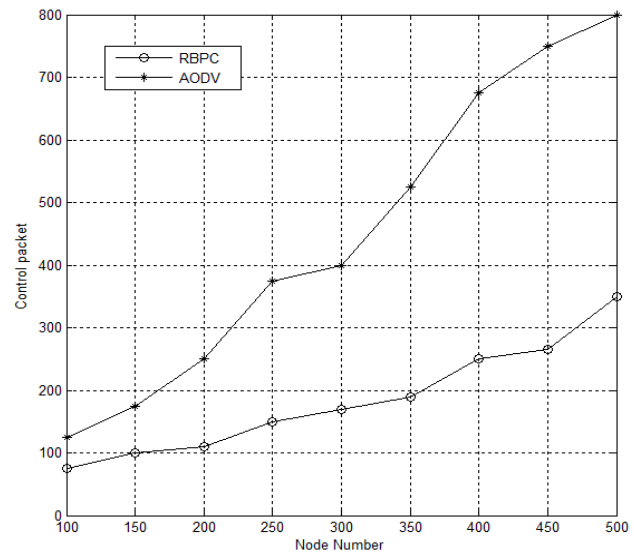

Figure 12. Control Group Performance

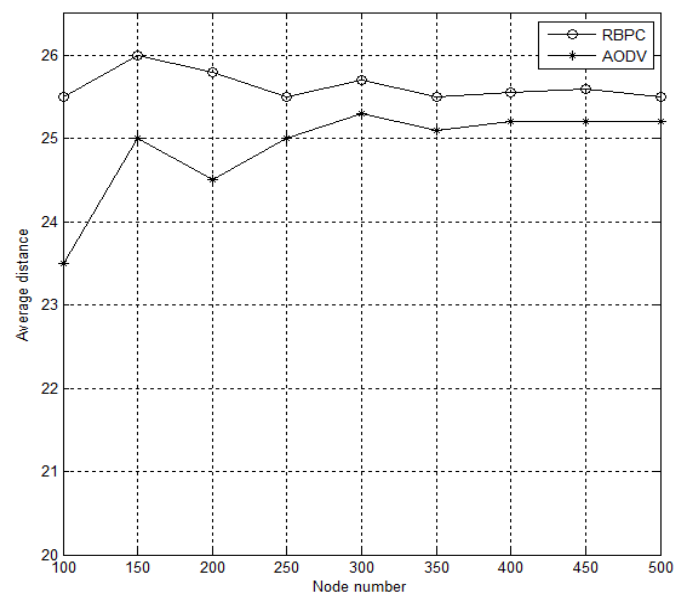

Figure 2. Overlapping Clusters and Cluster Scope

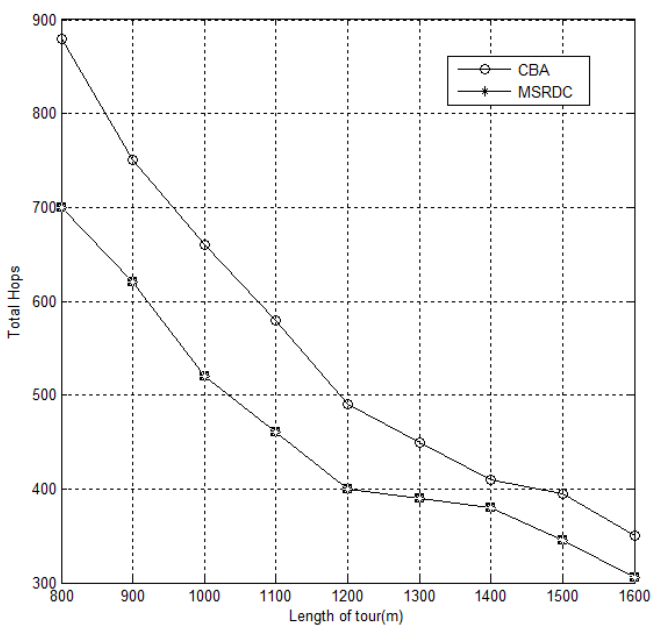

Figure 3. Network Transmission to the Total Number of Hops 


\section{Conclusion}

This paper first introduces the definition of routing metrics and the cognitive conditions needs consist of timing reference parameters. Then analyzed several kinds of typical routing metrics, lays the foundation for subsequent routing metrics definition. Finally consider cognitive wireless Ad hoc network has its unique characteristics, routing metrics should consider the use of primary user model; to guarantee the reliability of the cognitive user data transmission, the mutual interference between cognitive users should also be considered, therefore we define the routing metric to meet the needs of different end-to-end performance. Simulation results show that these routing parameters can accurately reflect the end-to-end performance of their own, and is better than by minimum hop routing performance.

\section{References}

[1] W. B. Heinzelman, A. P. Chandrakasan and H. Balakrishnan, "An application-specific protocol architecture for wireless micro sensor networks", IEEE Trans, on Wireless Communications, vol. 1, (2002) October, pp. $660-670$

[2] S. Cui, A. J. Goldsmith and A. Bahai, "Energy-efficiency of MIMO and cooperative MIMO techniques in sensor networks", IEEE Journal on Selected Areas in Communications, vol. 22, (2004), pp. 1089-1098.

[3] S. K. Jayaweera, "Virtual MIMO-based Cooperative Communication forEnergy-constrained Wireless Sensor Networks", IEEE Trans, on Wireless Communications, vol. 5, (2006) May, pp. 984-989.

[4] X. Li, M. Chen and W. Liu, "Application of STBC-encoded cooperative transmissions in wireless sensor networks", IEEE Signal Processing Letters, vol. 12, (2005), pp. 134-137.

[5] Y. Yuan, M. Chen and T. Kwon, "A novel cluster-based cooperative MIMO scheme for multi-hop wireless sensor networks", EURASIP Journal on Wireless Communications and Networking, vol. 2006, (2006), pp. 19.

[6] A. Paulraj, R. Nabar and D. Gore, "Introduction to Space-Time Wireless Communications", Cambridge University Press, Cambridge, U.K., (2003).

[7] S. K. Jayaweera, "Energy analysis of MIMO techniques in wireless sensor networks", In Proceedings of the 38th Annual Conference on Information Sciences and Systems (CISS), (2004) March.

[8] S. K. Jayaweera, "An energy-efficient virtual MIMO Communications architecture based on V-BLAST processing for distributed wireless sensor networks", Sensor and Ad Hoc Communications and Networks, 2004 First Annual IEEE Communications Society Conference on, (2004), pp. 299-308.

[9] M. Dohler, E. Lefranc and H. Aghvami, "Virtual antenna arrays for future wireless mobile communication systems", ICT 2002, Beijing, China, (2002) June.

[10] W. Zhang, H. He, Q. Zhang and T.-H. Kim, "Phone Protector: Protecting User Privacy on the Android-Based Mobile Platform", International Journal of Distributed Sensor Networks, vol. 2014, Article ID 282417, pp. 10 .

\section{Authors}

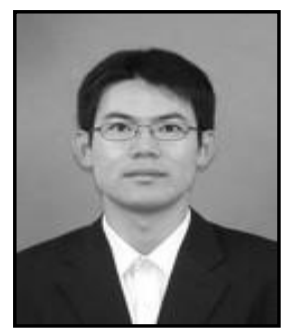

Han Tianfeng, (1981), male, Qingdao, Master, lecturer, the main research directions: Computer Application Technology. 
International Journal of Future Generation Communication and Networking Vol.7, No.4 (2014) 